\title{
The 2014 KCG meteor outburst: clues to a parent body
}

\author{
Althea V. Moorhead \\ NASA Meteoroid Environment Office, \\ Marshall Space Flight Center, Huntsville, Alabama 35812 \\ Peter G. Brown \\ Department of Physics and Astronomy, \\ The University of Western Ontario, London N6A3K7, Canada \\ Pavel Spurný \\ Astronomical Institute of the Academy of Sciences, \\ Fričova 298, CZ-251 65 Ondřejov Observatory, Czech Republic \\ William J. Cooke \\ NASA Meteoroid Environment Office, \\ Marshall Space Flight Center, Huntsville, Alabama 35812
}

\begin{abstract}
The $\kappa$ Cygnid (KCG) meteor shower exhibited unusually high activity in 2014, producing ten times the typical number of meteors. The shower was detected in both radar and optical systems and meteoroids associated with the outburst spanned at least five decades in mass. In total, the Canadian Meteor Orbit Radar, European Network, and NASA All Sky and Southern Ontario Meteor Network produced thousands of KCG meteor trajectories. Using these data, we have undertaken a new and improved characterization of the dynamics of this little-studied, variable meteor shower. The $\kappa$ Cygnids have a diffuse radiant and a significant spread in orbital characteristics, with multiple resonances appearing to play a role in the shower dynamics. We conducted a new search for parent bodies and found that several known asteroids are orbitally similar to the KCGs. $N$-body simulations show that the two best parent body candidates readily transfer meteoroids to the Earth in recent centuries, but neither produces an exact match to the KCG radiant, velocity, and solar longitude. We nevertheless identify asteroid 2001 MG1 as a promising parent body candidate.
\end{abstract}

